\title{
Politique patrimoniale et "patrimonialisation" aux Antilles françaises
}

\section{Thierry Nicolas}

\section{(2) OpenEdition \\ 1 Journals}

Édition électronique

URL : https://journals.openedition.org/tc/1903

DOI : $10.4000 /$ tc. 1903

ISSN : 1952-420X

Éditeur

Éditions de l'EHESS

\section{Édition imprimée}

Date de publication : 1 avril 2004

Pagination : 131-140

ISSN : 0248-6016

\section{Référence électronique}

Thierry Nicolas, «Politique patrimoniale et "patrimonialisation" aux Antilles françaises », Techniques \& Culture [En ligne], 42 | 2004, mis en ligne le 06 novembre 2007, consulté le 29 septembre 2022. URL: http://journals.openedition.org/tc/1903 ; DOI : https://doi.org/10.4000/tc.1903

Ce document a été généré automatiquement le 29 septembre 2022.

Tous droits réservés 


\title{
Politique patrimoniale et "patrimonialisation" aux Antilles françaises
}

\author{
Thierry Nicolas
}

1 Depuis trois décennies, les notions de protection et de défense du patrimoine occupent une place de plus en plus importante en Guadeloupe comme en Martinique. C'est d'abord l'État qui, dans le prolongement de la politique de conservation menée dans l'Hexagone, s'est attaché à partir de 1973 à inventorier, classer et restaurer l'héritage qui reflète le passé de ces îles. Puis, dans les années 1990, ce sont les insulaires qui se sont lancés dans un processus de "patrimonialisation » dans le but de sauvegarder des vestiges de leur histoire. Mais paradoxalement, ces deux dynamiques autour du patrimoine ne se superposent pas. Malgré d'évidentes similitudes, elles diffèrent dans leur logique intrinsèque. En effet, si elles se rapportent globalement à un même mouvement qui cherche à préserver et revaloriser les legs du passé, le nouvel intérêt des Antillais pour le patrimoine se distingue de la politique patrimoniale par un processus de réflexivité.

De la réflexivité de la patrimonialisation

2 La politique culturelle de l'État répond essentiellement à une conception du patrimoine largement inspirée des valeurs européennes intégrant la notion de valeur historique, de richesse et de beauté architecturale. Elle vise principalement, sous l'égide d'organismes étatiques divers telle que la Direction Régionale aux Affaires Culturelles (DRAC), à réhabiliter le patrimoine monumental. Cela va des édifices religieux (cathédrales, églises, presbytères, chapelles ou oratoires) aux bâtiments industriels (anciennes usines sucrières, distilleries ou habitations-distilleries). En matière de patrimoine civil, ce sont les anciens bâtiments de l'administration coloniale; en ce qui concerne le patrimoine militaire, ce sont les forts ou les batteries.

3 À la différence de la politique patrimoniale, la « patrimonialisation » ne se construit pas en considération du monument et de sa place dans la perspective de l'histoire et de l'art. Comme ont pu le démontrer D. Bégot et J.-M. Guibert (1996), les monuments et 
bâtiments, à l'exception des constructions industrielles, ne sont pas l'objet d'un intérêt particulier de la part des Antillais; car le patrimoine monumental (forts, bâtiments publics, habitations...) reste dans leur l'esprit lié au colonialisme. Confrontés de plus en plus systématiquement à l'Autre sous l'effet des transports aériens et maritimes ainsi que des moyens de communication à grande distance, ils voient dans le patrimoine ce gage de solidité qui leur permet de renforcer un enracinement spatial. Ils fouillent ainsi le passé afin d'y trouver les éléments qui leur confèreraient le statut d'élément symbolique qui fait « l'âme d'un pays ».

L'action autour du patrimoine idéel

4 L'un des domaines privilégiés de la logique de "patrimonialisation " insulaire s'avère être le patrimoine idéel, qui se rapporte à tous les héritages culturels non réalisés dans des objets concrets et palpables. La démarche de réflexivité fait de la langue le premier élément de ces legs historiques à protéger. Menacé par le phénomène de décréolisation ou de refrancisation, sa survie, pour beaucoup, n'est pas assurée. Ainsi, selon l'écrivain martiniquais Daniel Boukmann (1995) :

«Le créole, langue en danger de mort, a besoin d'assistance, et il ne faut pas que les générations à venir, de Martinique, de Guadeloupe, [...] nous accusent de ne pas avoir fait assez, même en français, pour la promotion de la langue créole. »

5 Les Guadeloupéens et les Martiniquais ne veulent pas que leur langue devienne une simple survivance folklorique, sorte de témoin du passé, comme cela peut être le cas dans l'Hexagone pour certaines langues régionales; ils souhaitent qu'elle soit une langue de culture à part entière. Dans cette optique, ils ont entrepris une série d'actions qui visent à ancrer le créole dans le temps long. Une attention particulière a ainsi été portée, notamment par les universitaires, à la nécessité d'inscrire cette langue orale dans l'écrit par la codification d'une graphie créole. Cela aboutit aujourd'hui à une situation où deux transcriptions à base phonologique sont désormais proposées. Cette stratégie de défense et de stabilisation de la langue passe également par la multiplication des démarches pour développer le nombre d'établissements scolaires qui proposent des enseignements de créole. Enfin, les Antillais ont milité pour l'inscription du créole dans la charte européenne des langues minoritaires régionales.

6 Ce travail de sauvetage et de préservation du créole conduit aussi à vouloir figer des éléments associés à cette langue. On pense en priorité aux proverbes et expressions d'antan mais surtout aux contes créoles, ces récits d'aventures fabuleux que l'on relatait aux enfants le soir. Ils ont disparu du quotidien antillais avec l'avènement de la télévision, et la patrimonialisation entend les promouvoir comme emblèmes de la richesse de l'imaginaire créole. La réalisation d'ouvrages, de CDs, de films, notamment des courts-métrages, l'organisation d'un festival du conte créole en Guadeloupe et des «nuits du conte créole » en Martinique sont devenus les supports principaux de cette réhabilitation.

7 D'autres legs idéels sont également remis en lumière. Les Antillais redécouvrent les musiques de percussion qui accompagnaient la vie quotidienne en milieu rural: le gwoka guadeloupéen et son homologue martiniquais le bel-air (bèlè), originaire du nord atlantique de l'île. Longtemps délaissées et connotées péjorativement, ces musiques se voient élevées en bannières d'une identité antillaise. Pour perpétuer la redécouverte et assurer la transmission du gwoka et du bèlè, de nombreuses associations œuvrent toute l'année à leur diffusion en dispensant des cours. Des écoles de danse et de musique existent maintenant dans presque toutes les communes des deux départements à Fort- 
de-France, Pointe-à-Pitre, Sainte-Anne, Rivière-Pilote, Sainte-Rose, Le François, etc. Ces associations organisent également des soirées comme les swaré bèlè et les léwoz ou des festivals, dont le fameux festival de gwoka de Sainte-Anne au mois de juillet qui connaît un succès retentissant (photo 1 ).

Le sursaut que connaissent ainsi les pratiques anciennes remet au goût du jour, à partir des témoignages de "personnes d'âge ", dépositaires de la mémoire de ces îles, des pratiques s'apparentant à des arts martiaux. En Martinique, c'est le danmyé, une sorte de combat cadencé rappelant la capoiera brésilienne qui sort de l'ombre ${ }^{1}$. En Guadeloupe, c'est le mayolé, dont on ne soupçonnait pas l'existence il y a encore deux décennies, que l'on essaie de relancer. Apparu à la fin du XVIII ${ }^{e}$ siècle, cet art martial qui oppose, au son du tambour, deux combattants armés de bâton avait presque disparu de la mémoire collective. Grâce à un groupe de mayoleurs basé au Moule, ce duel au bâton, qui s'apparente à la canne de combat, sort progressivement de la confidentialité dans laquelle l'avait plongé l'oubli.

Redécouvrir un patrimoine matériel et un savoir-faire

9 La «patrimonialisation » d'ordre matériel passe par la volonté de redécouvrir et de perpétuer les objets qui témoignent de la vie des prédécesseurs. On procède dans ce cadre à la reconstitution de villages anciens composés de cases traditionnelles que l'on trouvait dans les campagnes guadeloupéennes et martiniquaises dans la première moitié du XXe siècle et encore jusqu'aux années 1960. Il s'agit de reconsidérer ces logis sur la base d'un savoir-faire qui s'appuyait essentiellement sur l'utilisation de matériaux disponibles dans l'environnement naturel et de matériaux de récupération tels que les caisses de morues (photo 2).

Il n'est pas question d'avoir honte de ces cases de petite taille, n'excédant généralement pas une dizaine de mètres carrés, construites « artisanalement ». Au contraire, il s'agit de porter bien haut cette connaissance des ancêtres. C'est le cas de la case en gaulettes ${ }^{2}$, incarnant la case du « vyé nèg » (vieux nègre) qui se voit aujourd'hui élevée au rang de « construction » historique (photo 3 ).

11 Le désir de faire reconnaitre la valeur patrimoniale d'une architecture particulière s'exprime également dans la sauvegarde des maisons aux façades couvertes d'essentes, c'est-à-dire de planchettes de bois taillées dans des essences locales destinées à protéger les murs extérieurs en bois de l'humidité. Ces maisons en essentes, encore présentes sur la côte Sous-le-vent en Guadeloupe, bien que non classées, sont préservées par le renouvellement des essentes les plus usées (photo 4). 
Photo 1. Un « léwoz » avec Jomimi (crédit Brigitte Francius)

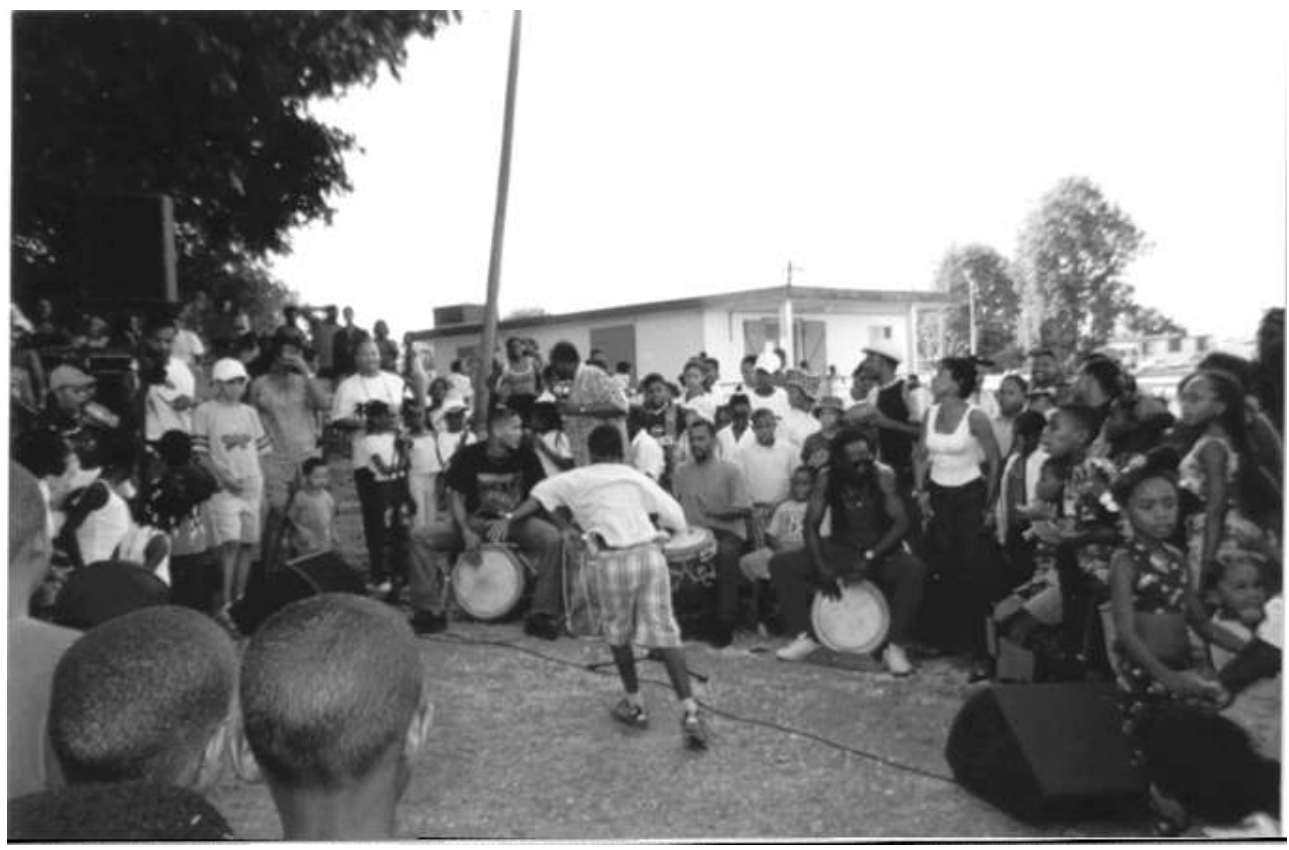

Photo 2. Reconstitution d'un village d'antan

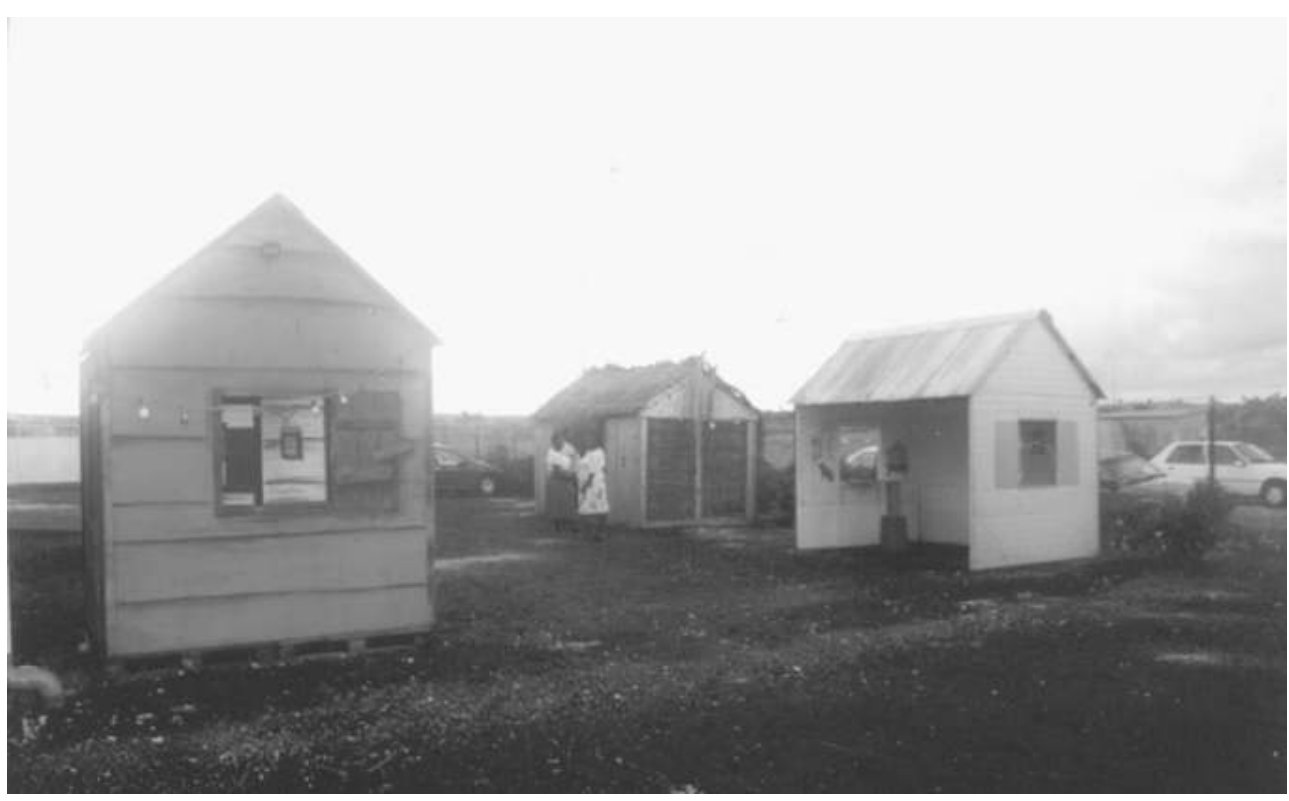




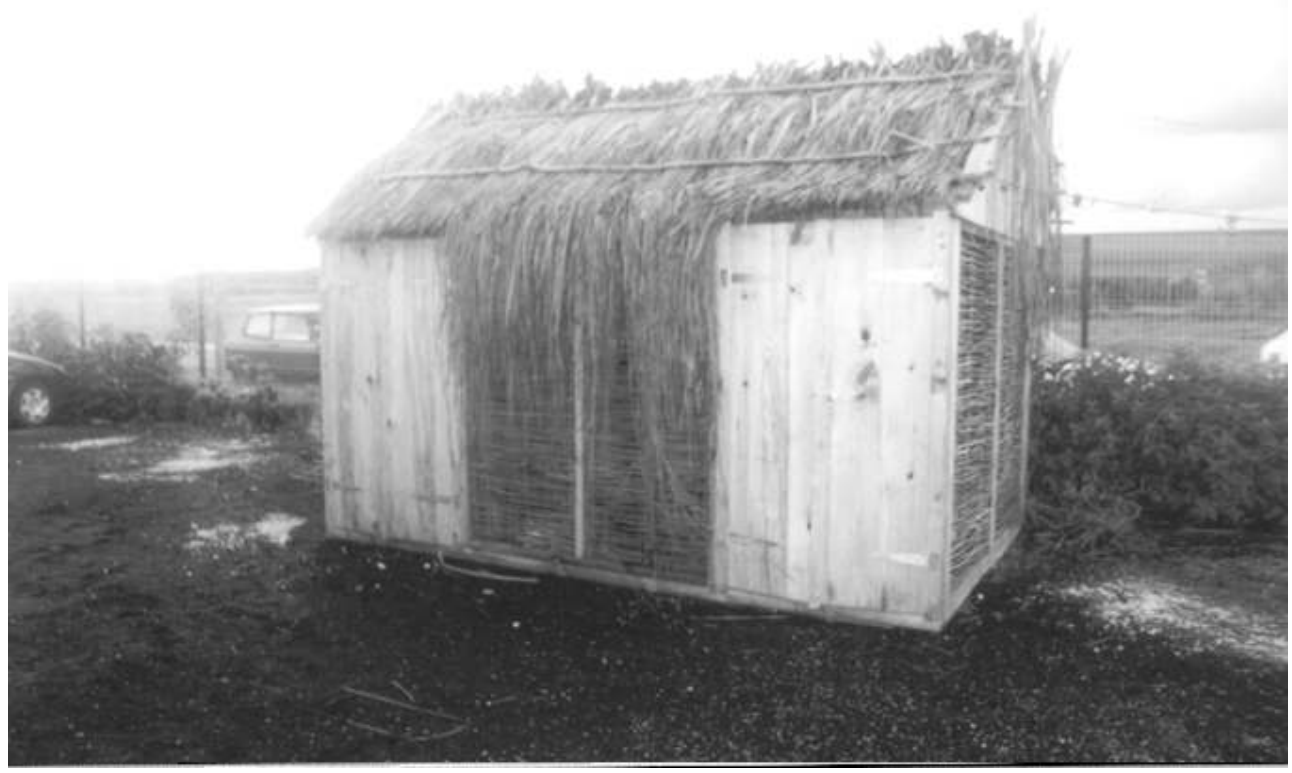

Photo 4. Une maison couverte d'essences

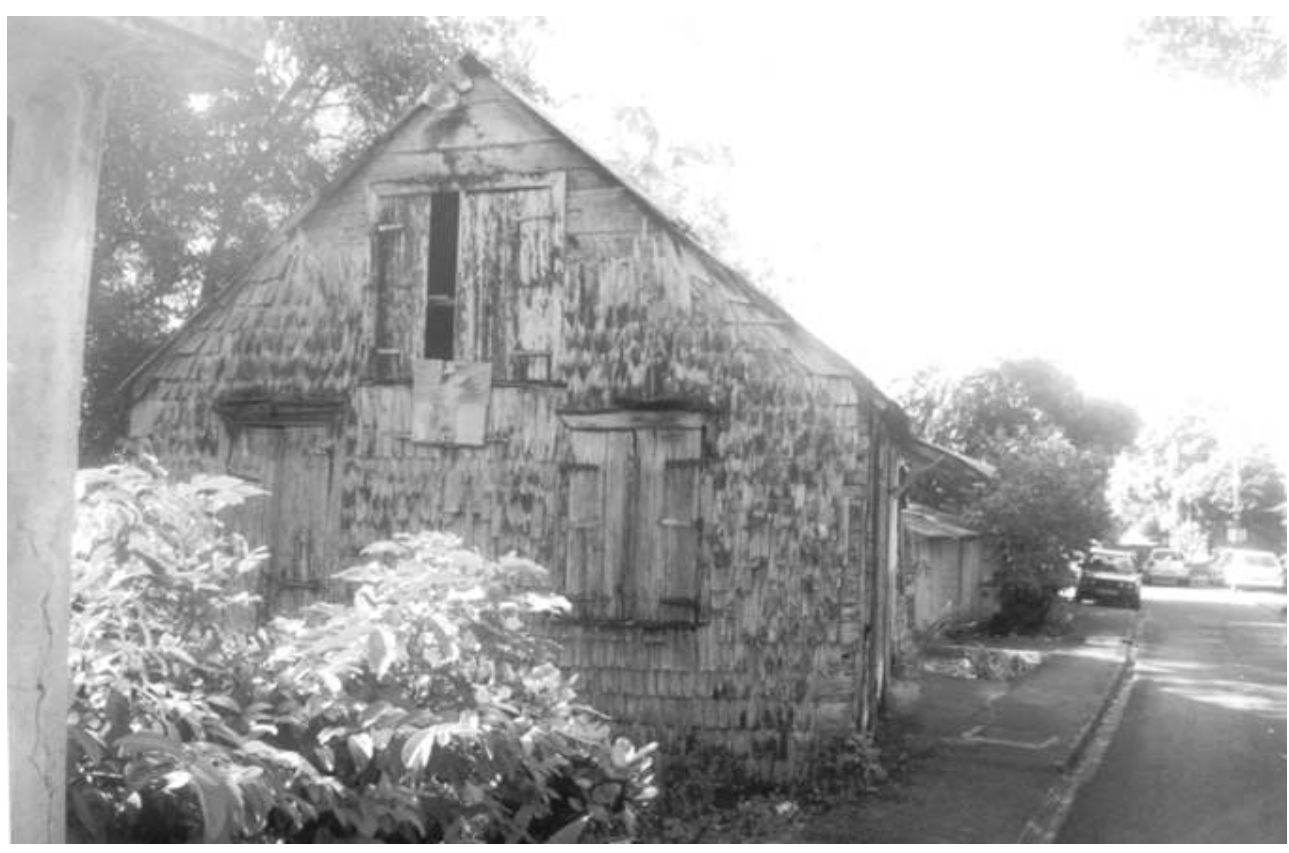

Cette volonté de restaurer un savoir-faire d'antan commande de redécouvrir les outils domestiques et les jouets que les enfants de cette génération ne connaissent plus. Car dans ce domaine, la transmission s'est interrompue avec l'élévation du niveau de vie et l'accroissement des importations. Ainsi essaie-t-on de relancer des ustensiles ou des accessoires de cuisine comme des pilons, des mortiers avec lesquels on broyait des aliments, des lélés, ces bâtons en bois qui servaient de mélangeur pour des préparations culinaires liquides. On réintroduit également les jouets, irrémédiablement surclassés par les divertissements importés, qui n'étaient ni industriels, ni artisanaux, mais réalisés spontanément à partir de prélèvements opérés dans la nature. Réapparaissent 
ainsi ces jouets dont la réalisation s'effectuait par imprégnation de savoirs pratiques et l'apprentissage par l'observation. On pense en particulier aux cerfs-volants dont les différentes variantes (kouli, vonvon...) étaient fabriquées à partir de lames de bambou ou de nervures de feuilles de coco pour l'armature, de feuilles d'arbre à pain ou de cacao pour le corps et d'une colle utilisant la moussache du manioc pour assembler le tout. C'est aussi le cas des kabwas (appelés encore tartines) qui sont des sortes de planches à roulettes, des trottinettes (photo 5) ou des toupies locales taillées au coutelas ou à la jambette (canif) dans un bois dur tel que le goyavier, le quénettier ou le citronnier.

Face à la litanie d'objets-témoins d'une période revalorisée, on pourrait encore multiplier les exemples. Cette revalorisation étant protéiforme et ne cessant de s'étendre à toutes sortes de domaines, il serait prétentieux de vouloir donner en dresser une liste exacte. Aussi l'énumération que nous pouvons en faire ici n'a-t-elle pas pour ambition l'exhaustivité, mais plutôt d'être indicative. Elle permet de se faire une idée de l'intérêt qui se centre sur les vestiges du passé, mais révèle surtout au grand jour l'action de ceux qui ont garanti leur continuité.

Les gardiens de la mémoire et l'émergence d'écomusées

La vaste entreprise dans laquelle se sont lancés les Guadeloupéens et les Martiniquais met en évidence l'action d'individus et de petites communautés, constituées généralement en associations, qui ont gardé et défendu la mémoire de ce patrimoine. Ils ont eu cette capacité unique à sauvegarder des supports de la mémoire menacés qui confortent les Antillais dans leur identité. De ce fait, la logique de patrimonialisation va de pair avec la volonté de consacrer les gardiens de la mémoire. Ceux qui ont maintenu contre vents et marées l'imaginaire de la langue créole, les rythmes des musiques de percussion, ou le savoir-faire des métiers d'autrefois sont l'objet d'un véritable culte. Les conteurs par exemple, après une longue éclipse, reviennent au premier plan et sont très demandés. Ils sont invités dans toutes sortes de manifestations, notamment dans les écoles et sur les plateaux de télévision (photo 6).

Photo 5. Une trottinette à l'ancienne

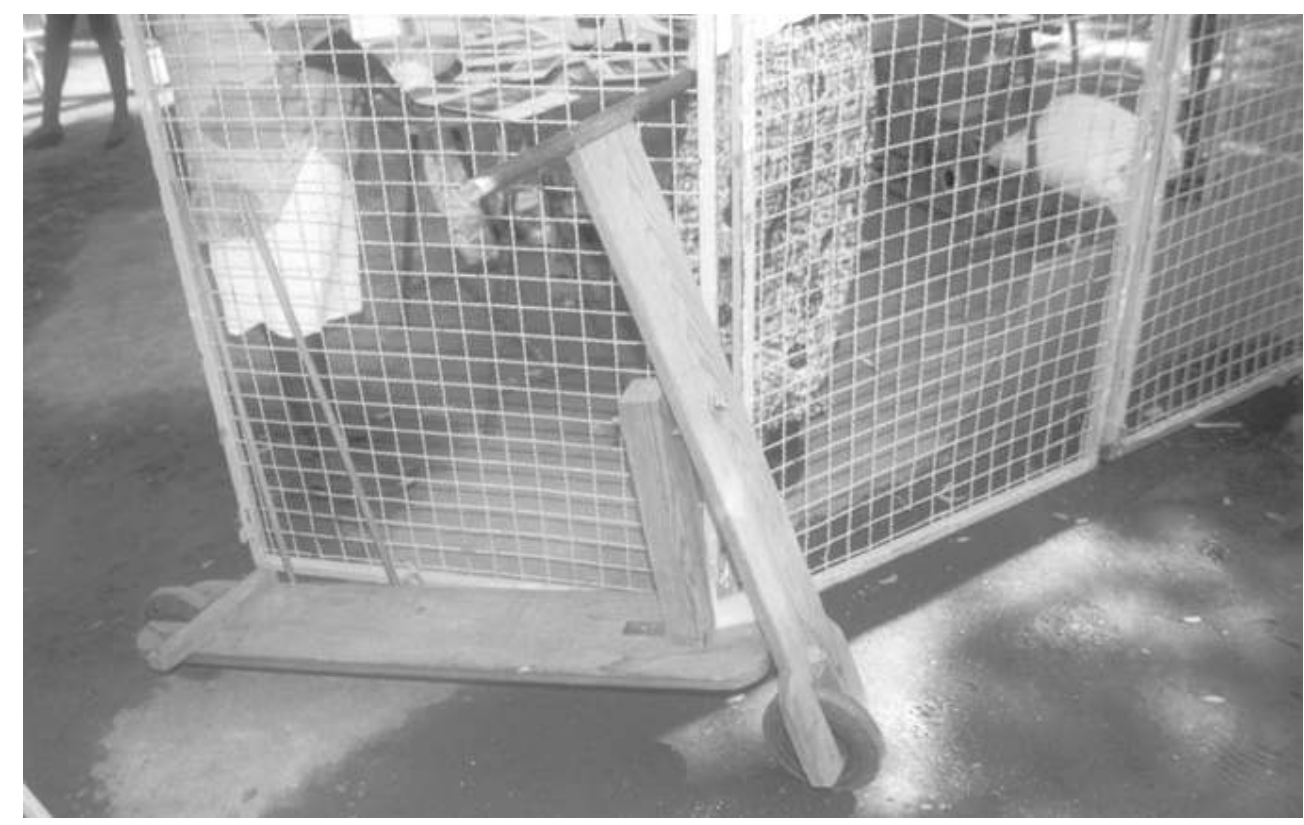


Photo 6. Un conteur dans une école primaire (Benzo) (Crédit Brigitte Francius)

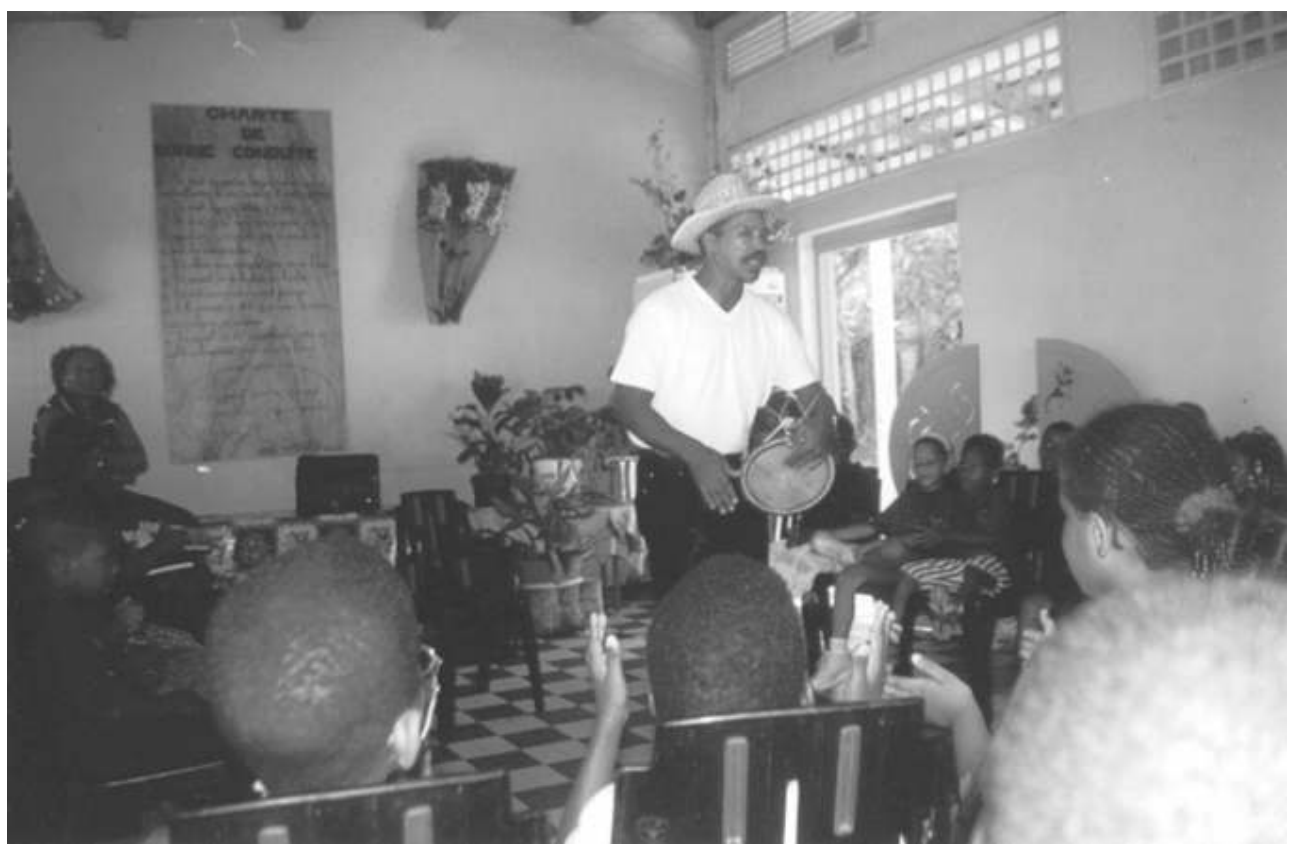

De même, les derniers représentants du métier peu connu de tailleur d'essentes sont assaillis de propositions visant à l'exposition de leur savoir-faire ancestral au cours de démonstrations (photo 7).

Ce devoir de mémoire conduit certains à créer des lieux où cet héritage pourrait être conservé et vu à rebours par les Antillais. Ils construisent des écomusées qui, comme l'a indiqué G-H Rivière, sont des miroirs « où les populations se regardent dans le but de se reconnaître » (1989). À Rivière-Pilote, à Pointe-Noire ou à Marie-Galante, ces écomusées offrent une sélection des legs du passé auxquels les Guadeloupéens et les Martiniquais peuvent s'identifier. Ils donnent à la patrimonialisation une signification dégagée du simple regard muséal que confère la politique patrimoniale étatique. Ces écomusées viennent manifestement rompre avec la dimension élitiste et le manque de réflexivité des musées traditionnels. 
Photo 7. Un tailleur d'essentes (Benjamin Kamoise)

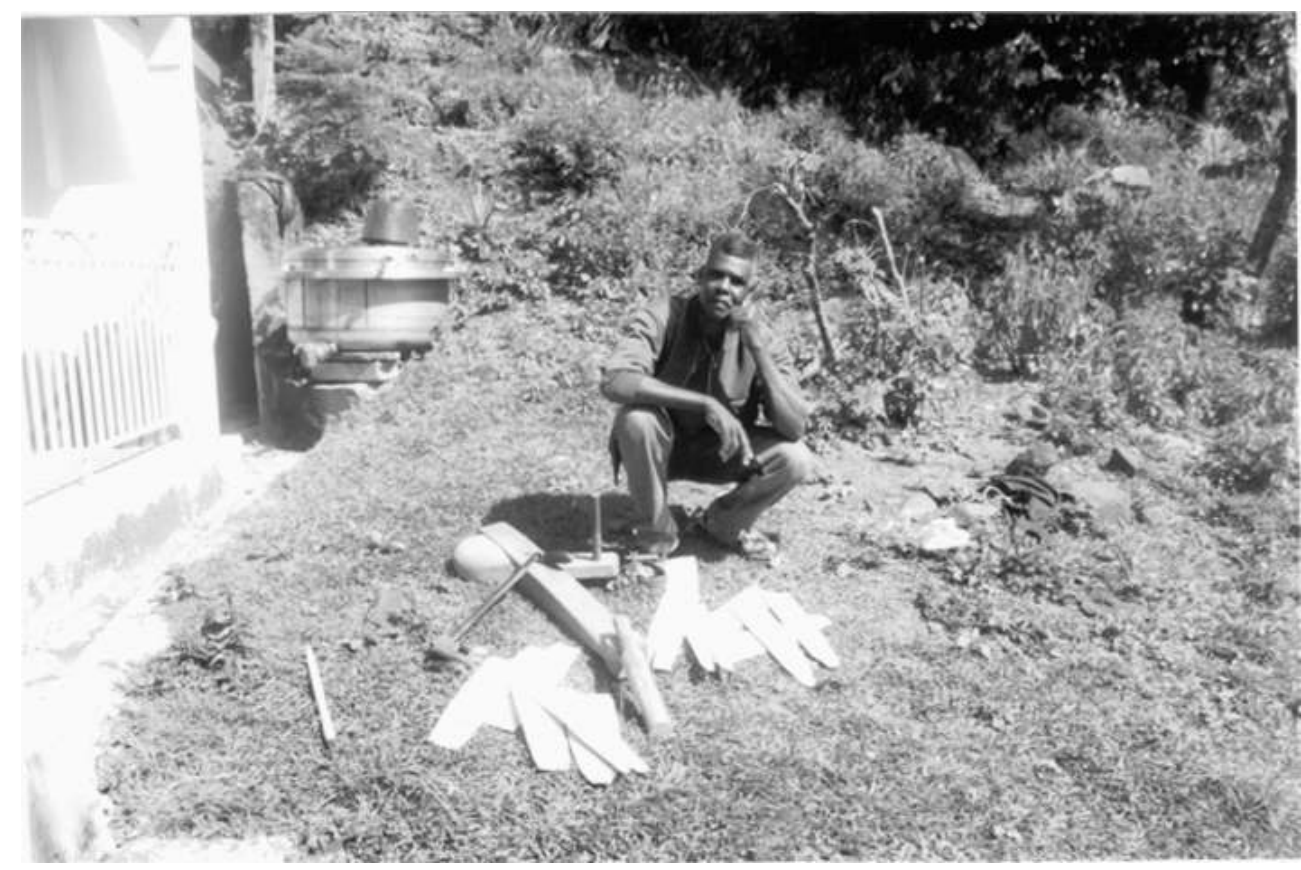

17 Somme toute, nous avons essayé de montrer la spécificité du processus de «patrimonialisation» mené par les Antillais depuis les années 1990. Ce regard particulier sur le patrimoine les pousse à mettre en avant, avec fierté, une connaissance relativement empirique face à une logique institutionnelle qui privilégie les bâtisses les plus prestigieuses. Leur principal but est de faire émerger de vieilles coutumes, des objets ou des outils usuels plutôt que de demander le classement de tel ou tel bâtiment. Toutefois, ce travail de résurrection des héritages du passé ne peut empêcher la disparition de certains éléments comme les chants de travail qui rythmaient l'effort des hommes afin d'accroître la productivité durant la période esclavagiste. Ni les chants des sarcleurs, ni les chants des scieurs de long ou des dameurs de rues n'ont résisté à l'épreuve du temps et de l'oubli. Ainsi, la " patrimonialisation » ne permet pas de tout redécouvrir et doit faire face à une rupture définitive de la "chaîne de transmission ». Chaque jour davantage, les Antillais doivent composer avec ces disparitions irréversibles.

\section{BIBLIOGRAPHIE}

Bégot, D. \& J.-M. Guibert

1996. " Archéologie industrielle et restauration en milieu tropical : un exemple, la Guadeloupe. », in Les musées des départements français d'Amérique, Actes du congrès de l'International Council of Museums, 14-18 novembre 1994. Fort-de-France.

Boukman, D.

1995. « Société et littérature antillaises aujourd'hui », Cahiers de l'Université de Perpignan 25. 
Rivière, Georges-Henri

1989. La Muséologie selon Georges-Henri Rivière : cours de muséologie, textes et témoignages. Paris :

Dunod.

\section{NOTES}

1. On retrouve également le danmyé sous l'appellation de ladja ou de kokoyé. Provenant des danses initiatiques africaines, il a été réaménagé dans le contexte des habitations. Le danmyé oppose deux lutteurs qui associent frappes et saisies au rythme du tambour à l'intérieur d'un cercle formé par les spectateurs, le lawonn.

2. Ces cases sont constituées de parois sous formes de panneaux de gaulettes, dont elles tirent leur nom, qui sont souvent des petites branches robustes et flexibles de bois tibaume entrelacées. Dans certains cas, cette ossature est couverte par un torchis à base de bouse de vache ou de boue mélangée à de la paille de canne. La toiture qui repose sur des lattes en bois dur (acomat, poirier, abricotier...) est généralement constituée de feuilles séchées (têtes de canne, rameaux ou feuilles de palmistes).

\section{AUTEUR}

\section{THIERRY NICOLAS}

Laboratoire Espace et Culture (Université Paris IV- CNRS) 